\title{
Growth and fixed points of meromorphic solutions of nonhomogeneous linear differential equations
}

Junfeng $\mathrm{Xu}^{1,2^{*}}$ and Zhanliang Zhang ${ }^{3}$

\section{"Correspondence:}

xujunf@gmail.com

1 Department of Mathematics,

Hunan University, Changsha,

410082, P.R. China

${ }^{2}$ Department of Mathematics, Wuyi

University, Jiangmen, 529020,

P.R. China

Full list of author information is

available at the end of the article

\begin{abstract}
In this paper, we investigate the growth and fixed points of meromorphic solutions and their derivatives of higher-order nonhomogeneous linear differential equations with meromorphic coefficients. Our results extend the previous theorems due to Gundersen and Yang, Han and Yi. As an application of our results, we generalized some previous results that are related to Brück's conjecture.
\end{abstract}

MSC: Primary 34M10; secondary 30D35

Keywords: meromorphic function; growth order; fixed point

\section{Introduction and main results}

In this paper, we shall assume that the reader is familiar with the fundamental results and the standard notations of the Nevanlinna value distribution theory of meromorphic functions (see [1-3]). The term 'meromorphic function' will mean meromorphic in the whole complex plane $\mathbb{C}$. In addition, we will use notations $\rho(f)$ to denote the order of growth of a meromorphic function $f(z), \lambda(f)$ to denote the exponents of convergence of the zerosequence of a meromorphic function $f(z), \bar{\lambda}(f)$ to denote the exponents of convergence of the sequence of distinct zeros of $f(z)$.

Let $f(z), g(z)$ and $a(z)$ be three meromorphic functions with that $f(z)$ and $g(z)$ are nonconstant. We say that $f(z)$ and $g(z)$ share $a(z)$ CM (counting multiplicities) if $f(z)-a(z)$ and $g(z)-a(z)$ have the same zeros with the same multiplicities.

In order to give some estimates of fixed points, we recall the following definitions (see $[4,5])$.

Definition 1.1 Let $z_{1}, z_{2}, \ldots\left(\left|z_{j}\right|=r_{j}, 0 \leq r_{1} \leq r_{2} \leq \cdots\right)$ be the sequence of distinct fixed points of transcendental meromorphic function $f$. Then $\bar{\tau}(f)$, the exponent of convergence of the sequence of distinct fixed points of $f$, is defined by

$$
\bar{\tau}(f)=\inf \left\{\tau>\left.0\left|\sum_{j=1}^{\infty}\right| z_{j}\right|^{-\tau}<+\infty\right\} .
$$

It is evident that $\bar{\tau}(f)=\varlimsup_{r \rightarrow \infty} \frac{\log \bar{N}\left(r, \frac{1}{f-z}\right)}{\log r}$ and $\bar{\tau}(f)=\bar{\lambda}(f-z)$.

Non-homogeneous linear differential equations is an important area of complex differential equations, which has a large number of potential applications. For growth estimates 
of solutions of a non-homogeneous linear differential equation, in general there exist exceptional solutions that are not easy to discuss (see [6], Chapter 8).

Gundersen and Yang [7] proved the following well-known result which can guarantee that every solution of non-homogeneous linear differential equations has infinite order.

Theorem A Let P be a nonconstant polynomial. Then every solution $f$ of the differential equation $f^{\prime}-e^{P(z)} f=1$ is an entire function of infinite order.

Remark 1.1 Yang generalized the above result to $f^{(k)}$ in [8].

Han and Yi [9] improved Theorem A and obtained the following.

Theorem B Let $P(z)$ be a nonconstant polynomial, $Q(z)(\not \equiv 0)$ be a polynomial. Then every solution $f \not \equiv$ of the equation

$$
f^{\prime}-e^{P(z)} f=Q(z)
$$

has infinite order.

Lü, Liu and Yi [10] also generalized Theorem B and proved the following result.

Theorem C Let $P(z), R(z)(\not \equiv 0), Q(z)(\not \equiv 0)$ be three polynomials with that $P(z)$ is nonconstant. Then every solution $f \not \equiv 0$ of the equation

$$
f^{\prime}-R(z) e^{P(z)} f=Q(z)
$$

has infinite order.

In this paper, we generalized the above results by a different argument.

Theorem 1.1 Let $P(z)$ be a nonconstant polynomial, $R(z)(\not \equiv 0), Q(z)(\not \equiv 0)$ be meromorphic functions with $\max \{\rho(R), \rho(Q)\}<\operatorname{deg}(P)=n$ and $k$ be a positive integer. Then every meromorphic solution $f \not \equiv 0$, whose poles are of uniformly bounded multiplicities, of the equation

$$
f^{(k)}-R(z) e^{P(z)} f=Q(z)
$$

has infinite order and satisfies $\bar{\lambda}(f)=\lambda(f)=\rho(f)=\infty$.

Corollary 1.2 Let $P(z)$ be a nonconstant polynomial, $R(z)(\not \equiv 0), Q(z)(\not \equiv 0)$ be meromorphic functions that have finitely many poles and $\max \{\rho(R), \rho(Q)\}<\operatorname{deg}(P)=n$ and $k$ be a positive integer. Then every meromorphic solution $f \not \equiv 0$ of equation (1.3) has infinite order and satisfies $\bar{\lambda}(f)=\lambda(f)=\rho(f)=\infty$.

Corollary 1.3 Let $P(z)$ be a nonconstant polynomial, $R(z)(\not \equiv 0), Q(z)(\not \equiv 0)$ be rational functions and $k$ be a positive integer. Then every meromorphic solution $f \not \equiv$ of equation (1.3) has infinite order and satisfies $\bar{\lambda}(f)=\lambda(f)=\rho(f)=\infty$. 
Since the beginning of the last four decades, a substantial number of research articles have been written to describe the fixed points of general transcendental meromorphic functions (see [11]). However, there are few studies on the fixed points of solutions of the differential equation. As shown in [4], Chen first studied the problems on the fixed points of solutions of second-order linear differential equations with entire coefficients. Since then, Wang and Yi [8], Laine and Rieppo [12], Chen and Shon [13], Liu and Zhang [14], Xu and Yi [15] studied the problems on the fixed points of solutions of homogeneous linear differential equations with meromorphic coefficients and their derivatives. The other main purpose of this paper is to investigate the fixed point of the solution of (1.3).

Theorem 1.4 Let $P(z), R(z), Q(z)$ satisfy the additional hypotheses of Theorem 1.1. If $\not \equiv 0$ is any meromorphic solution, whose poles are of uniformly bounded multiplicities, of equation (1.3), then $f, f^{\prime}, f^{\prime \prime}$ all have infinitely many fixed points and satisfy

$$
\bar{\tau}(f)=\bar{\tau}\left(f^{\prime}\right)=\bar{\tau}\left(f^{\prime \prime}\right)=\infty .
$$

\section{Lemmas}

The linear measure of a set $E \subset[0,+\infty)$ is defined as $m(E)=\int_{0}^{+\infty} \chi_{E}(t) d t$. The logarithmic measure of a set $E \subset[1,+\infty)$ is defined by $\operatorname{lm}(E)=\int_{1}^{+\infty} \chi_{E}(t) / t d t$, where $\chi_{E}(t)$ is the characteristic function of $E$.

Lemma 2.1 [16] Letf be a transcendental meromorphic function of finite order $\sigma$. Let $\varepsilon>0$ be a constant, and $k$ and $j$ be integers satisfying $k>j \geq 0$. Then the following two statements hold:

(a) There exists a set $E_{1} \subset(1, \infty)$ which has finite logarithmic measure such that for all $z$ satisfying $|z| \notin E_{1} \cup[0,1]$, we have

$$
\left|\frac{f^{(k)}(z)}{f^{(j)}(z)}\right| \leq|z|^{(k-j)(\sigma-1+\varepsilon)}
$$

(b) There exists a set $E_{2} \subset[0,2 \pi)$ which has linear measure zero such that if $\theta \in[0,2 \pi)-E_{2}$, then there is a constant $R=R(\theta)>0$ such that (2.1) holds for all $z$ satisfying $\arg z=\theta$ and $R \leq|z|$.

Lemma 2.2 [15] Let $f(z)=g(z) / d(z)$, where $g(z)$ is transcendental entire, and let $d(z)$ be the canonical product (or polynomial) formed with the nonzero poles of $f(z)$. Then we have

$$
\begin{aligned}
f^{(n)} & =\frac{1}{d}\left[g^{(n)}+B_{i, i-1} g^{(n-1)}+\cdots+B_{i, 1} g^{\prime}+B_{i, 0} g\right], \\
\frac{f^{(n)}}{f} & =\left[\frac{g^{(n)}}{g}+B_{i, i-1} \frac{g^{(n-1)}}{g}+\cdots+B_{i, 1} \frac{g^{\prime}}{g}+B_{i, 0}\right],
\end{aligned}
$$

where $B_{i, j}$ are defined as a sum of a finite number of terms of the type

$$
\sum_{\left(j_{1} \cdots j_{i}\right)} C_{i j_{1} \cdots j_{i}}\left(\frac{d^{\prime}}{d}\right)^{j_{1}} \cdots\left(\frac{d^{(i)}}{d}\right)^{j_{i}},
$$

$C_{j j_{1} \cdots j_{i}}$ are constants, and $j+j_{1}+2 j_{2}+\cdots+i j_{i}=n$. 
Using mathematical induction, we can easily prove the lemma.

Lemma 2.3 [17] Let $g(z)$ be a transcendental meromorphic function with $\sigma(g)=\beta<\infty$. Then, for any given $\varepsilon>0$, there exists a set $E \subset[0,2 \pi)$ that has linear measure zero such that if $\psi \in[0,2 \pi) \backslash E$, then there is a constant $R=R(\psi)>1$ such that, for all $z$ satisfying $\arg z=\psi$ and $|z|=r>R$, we have

$$
\exp \left\{-r^{\beta+\varepsilon}\right\} \leq|g(z)| \leq \exp \left\{r^{\beta+\varepsilon}\right\}
$$

Lemma 2.4 [6] Let $P(z)=(\alpha+i \beta) z^{n}+\cdots(\alpha, \beta$ are real, $|\alpha|+|\beta| \neq 0)$ be a polynomial with degree $n \geq 1, A(z) \not \equiv 0$ be a meromorphic function with $\sigma(A)<n$. Set $g(z)=A(z) e^{P(z)}$, $z=r e^{i \theta}, \delta(P, \theta)=\alpha \cos n \theta-\beta \sin n \theta$, then for any given $\varepsilon>0$, there is a set $E_{1} \subset[0,2 \pi)$ that has linear measure zero such that for any $\theta \in[0,2 \pi) \backslash\left(E_{1} \cup E_{2}\right)$ and a sufficiently large $r$, we have:

(i) If $\delta(P, \theta)>0$, then

$$
\exp \left\{(1-\varepsilon) \delta(P, \theta) r^{n}\right\} \leq\left|g\left(r e^{i \theta}\right)\right| \leq \exp \left\{(1+\varepsilon) \delta(P, \theta) r^{n}\right\} ;
$$

(ii) If $\delta(P, \theta)<0$, then

$$
\exp \left\{(1+\varepsilon) \delta(P, \theta) r^{n}\right\} \leq\left|g\left(r e^{i \theta}\right)\right| \leq \exp \left\{(1-\varepsilon) \delta(P, \theta) r^{n}\right\},
$$

where $E_{2}=\{\theta \in[0,2 \pi) ; \delta(P, \theta)=0\}$ is a finite set.

Lemma 2.5 [18] Let $A_{0}, A_{1}, \ldots, A_{k-1}, F \not \equiv 0$ be meromorphic functions offinite order. Iff $(z)$ is an infinite-order meromorphic solution of the equation

$$
f^{(k)}+A_{k-1} f^{(k-1)}+\cdots+A_{1} f^{\prime}+A_{0} f=F,
$$

then $f$ satisfies $\lambda(f)=\bar{\lambda}(f)=\sigma(f)=\infty$.

Lemma 2.6 [19] Let $f(z)$ be an entire function, and suppose that

$$
G(z):=\frac{\log ^{+} f^{(k)}(z)}{|z|^{\rho}}
$$

is unbounded on some ray $\arg z=\theta$ with a constant $\rho>0$, then there exists an infinite sequence of $z_{m}=r_{m} e^{i \theta}$, where $r_{m} \rightarrow \infty$, such that $G\left(z_{m}\right) \rightarrow \infty$.

Lemma 2.7 (see [19]) Let $g(z)$ be an entire function with $\rho(g)=\rho<\infty$. Suppose that there exists a set $H \subset[0,2 \pi)$ which has linear measure zero such that $\log ^{+}\left|f\left(r e^{i \theta}\right)\right| \leq M r^{\rho}$ for any ray $\arg z=\theta \in[0,2 \pi) \backslash H$, where $M$ is a positive constant depending on $\theta$, where $M$ is a positive constant independent of $\theta$. Then $\rho(g) \leq \rho$.

The next lemma plays a key role in our proof of Theorem 1.1, some ideas are due to [19-21]. 
Lemma 2.8 Let $P(z)$ be a nonconstant polynomial, $R(z)(\not \equiv 0), Q(z)(\not \equiv 0)$ be meromorphic functions with $\max \{\rho(R), \rho(Q)\}<\operatorname{deg}(P)=n$ and $k$ be a positive integer. We denote

$$
L_{f}=f^{(k)}-R(z) e^{P(z)} f
$$

If $\not \equiv 0$, whose poles are of uniformly bounded multiplicities, is a finite-order meromorphic solution of $(1.3)$, then $\rho\left(L_{f}\right) \geq n$.

Proof Suppose that $\rho\left(L_{f}\right)<n$, we consider two cases.

(i) If $\rho(f)<n$, then $\rho\left(f^{(k)}\right)<n$. $L_{f}=f^{(k)}-R(z) e^{P(z)} f$ has the form

$$
R(z) e^{P(z)}=\frac{L_{f}-f^{(k)}}{f}
$$

Note that $\rho\left(\operatorname{Re}^{P}\right)=n$ and $\rho\left(\frac{L_{f}-f^{(k)}}{f}\right)<n$, we can obtain a contradiction.

(ii) If $n \leq \rho(f)<\infty$, we rewrite (2.2) into

$$
\frac{L_{f}}{f}=\frac{f^{(k)}}{f}+R(z) e^{P(z)}
$$

From equation (1.3), we know that the poles of $f(z)$ can occur only at the poles of $R(z)$, $Q(z)$. Note that the multiplicities of pole points of $f$ are uniformly bounded, and thus we have

$$
N(r, f) \leq M \bar{N}(r, f) \leq M \max \{N(r, R), N(r, Q)\},
$$

where $M$ is a positive constant. Then we have $\lambda(1 / f) \leq \alpha=\max \{\rho(R), \rho(Q)\}<n$.

Let $f=g / d, d$ be the canonical product formed with the nonzero poles of $f(z)$, with $\beta=\rho(d)=\lambda(d)=\lambda(1 / f) \leq \alpha<n, g$ be an entire function and $n \leq \rho(g)=\rho(f)=\rho<\infty$. Substituting $f=g / d$ into (2.3), by Lemma 2.2 we can get

$$
\frac{g^{(k)}}{g}+\frac{g^{(k-1)}}{g} B_{k, k-1}+\cdots+\frac{g^{\prime}}{g} B_{i, 1}+B_{i, 0}+\operatorname{Re}^{P}=\frac{d L_{f}}{g} .
$$

By Lemma 2.1, for any given $\varepsilon(0<\varepsilon<n-\beta)$, there exists a set $E_{0} \in[0,2 \pi)$ that has linear measure zero such that if $\theta \in[0,2 \pi) \backslash E_{0}$, then there is a constant $R_{0}=R_{0}(\theta)>1$ such that for all $z$ satisfying $\arg z=\theta$ and $|z| \geq R_{0}$, we have

$$
\left|\frac{g^{(j)}(z)}{g(z)}\right| \leq|z|^{k(\rho-1+\varepsilon)} \quad(j=1,2, \ldots, k)
$$

and

$$
\left|\frac{d^{(j)}(z)}{d(z)}\right| \leq|z|^{k(\beta-1+\varepsilon)} \quad(j=1,2, \ldots, k) .
$$

Set $\rho\left(L_{f}\right)=\gamma<n$. Then, by Lemma 2.3, for the above given $\varepsilon(0<2 \varepsilon<\min \{n-\gamma, n-\beta\})$, there exists a set $E_{1} \subset[0,2 \pi)$ that has linear measure zero such that if $\theta \in[0,2 \pi) \backslash E_{1}$, then 
there is a constant $R=R(\theta)>1$ such that, for all $z$ satisfying $\arg z=\theta$ and $|z|=r>R$, we have, for sufficiently large $r$,

$$
\left|L_{f}(z)\right| \leq \exp \left\{r^{\gamma+\varepsilon}\right\} \text {. }
$$

By Lemma 2.4, there exists a ray $\arg z=\theta \in[0,2 \pi) \backslash\left(E_{0} \cup E_{1} \cup E_{2}\right), E_{2}=\{\theta \in$ $[0,2 \pi) ; \delta(P, \theta)=0\}$ being a finite set such that $\delta(P, \theta)>0$, and for the above given $\varepsilon$, when $r$ is sufficiently large, we have

$$
\left|R\left(r e^{i \theta}\right) e^{P\left(r e^{i \theta}\right)}\right| \geq \exp \left\{(1-\varepsilon) \delta(P, \theta) r^{n}\right\} .
$$

We now prove that

$$
\log ^{+}|g(z)| /|z|^{\beta+\varepsilon}
$$

is bounded on the ray $\arg z=\theta$. We assume that

$$
\log ^{+}|g(z)| /|z|^{\beta+\varepsilon}
$$

is unbounded on the ray $\arg z=\theta$. Then, by Lemma 2.6, there is a sequence of points $z_{m}=r_{m} e^{i \theta}$ such that $r_{m} \rightarrow+\infty$ and that

$$
\frac{\log ^{+}\left|g\left(z_{m}\right)\right|}{r_{m}^{\beta+\varepsilon}} \rightarrow+\infty
$$

From (2.7) and (2.9), we get

$$
\frac{\left|d\left(z_{m}\right) L_{f}\left(z_{m}\right)\right|}{\left|g\left(z_{m}\right)\right|} \rightarrow 0
$$

for $m$ is large enough.

By (2.4)-(2.6), (2.8) and (2.10), we obtain

$$
\begin{aligned}
\exp \left\{(1-\varepsilon) \delta(P, \theta) r_{m}^{n}\right\} \leq & \left|R\left(r_{m} e^{i \theta}\right) e^{P\left(r_{m} e^{i \theta}\right)}\right| \\
\leq & \left|\frac{g^{(k)}}{g}+\frac{g^{(k-1)}}{g} B_{k, k-1}+\cdots+\frac{g^{\prime}}{g} B_{i, 1}+B_{i, 0}\right|+\left|\frac{\left|d\left(r_{m}\right) L_{f}\left(r_{m}\right)\right|}{\left|g\left(r_{m}\right)\right|}\right| \\
\leq & r_{m}^{k(\sigma-1+\varepsilon)}+r_{m}^{(k-1)(\sigma-1+\varepsilon)} M r_{m}^{k(\beta-1+\varepsilon)}+\cdots \\
& +M r_{m}^{k(\beta-1+\varepsilon)}+\exp \left\{r_{m}^{\beta+\varepsilon}\right\} \leq M \exp \left\{r_{m}^{\beta+2 \varepsilon}\right\},
\end{aligned}
$$

where $M(0<M<\infty)$ is a constant and $M$ may be different at different places. It is a contradiction with $\beta+2 \varepsilon<n$. Hence

$$
\log ^{+}|g(z)| /|z|^{\beta+\varepsilon}
$$

is bounded on the ray $\arg z=\theta$. This is, $|g(z)| \leq M \exp \left\{r^{\beta+\varepsilon}\right\}$ on the ray $\arg z=\theta$. Then, by Lemma 2.7, we have $\rho(g) \leq \beta+\varepsilon<n$, this is a contradiction.

Hence $\rho\left(L_{f}\right) \geq n$.

The proof of Lemma 2.8 is completed. 


\section{Proof of Theorem 1.1}

Assume that $f(\not \equiv 0)$ is a meromorphic function of (1.3). Suppose, to the contrary, that $\rho(f)<\infty$. By Lemma 2.8, we have $n \leq \rho\left(L_{f}\right)=\rho(Q)<n$ and this is a contradiction. Hence every solution $f$ of equation (1.3) is of infinite order. By Lemma 2.5, every solution $f$ of equation (1.3) satisfies $\bar{\lambda}(f)=\lambda(f)=\rho(f)=\infty$. The proof of Theorem 1.1 is completed.

\section{Proof of Theorem 1.4}

Assume that $f(\not \equiv 0)$ is a meromorphic function of (1.3), then by Theorem 1.1 we have $\rho(f)=\infty$. Set $g_{0}(z)=f(z)-z$, then $z$ is a fixed point of $f(z)$ if and only if $g_{0}(z)=0 . g_{0}(z)$ is a meromorphic function and $\rho\left(g_{0}\right)=\rho(f)=\infty$. If $k \geq 2$, substituting $f=g_{0}+z, f^{(k)}=g_{0}^{(k)}$ into (1.3), we have

$$
g_{0}^{(k)}-\operatorname{Re}^{P} g_{0}=Q-z R e^{P} .
$$

Obviously, $Q-z R e^{P} \not \equiv 0$. Here we just consider the meromorphic solutions of infinite order satisfying $g_{0}=f-z$, by Lemma 2.5 we know that $\bar{\lambda}\left(g_{0}\right)=\bar{\tau}(f)=\infty$ holds. If $k=1$, substituting $f=g_{0}+z, f^{\prime}=g_{0}^{\prime}+1$ into (1.3), we can also obtain the same conclusion by a similar way.

Now we consider the fixed points of $f^{\prime}(z)$.

Let $g_{1}(z)=f^{\prime}-z$, then $z$ is a fixed point of $f^{\prime}(z)$ if and only if $g_{1}(z)=0 . g_{1}(z)$ is a meromorphic function and $\rho\left(g_{1}\right)=\rho\left(f^{\prime}\right)=\rho(f)=\infty$. Differentiating both sides of equation (1.3), we have

$$
f^{(k+1)}-R e^{P} f^{\prime}-\left(R^{\prime}+R P^{\prime}\right) e^{P} f=Q^{\prime}
$$

By (1.3), we have

$$
f=-\frac{1}{R e^{P}}\left[f^{(k)}-Q\right]
$$

Substituting (4.3) into (4.2), we derive

$$
f^{(k+1)}-\left[\frac{R^{\prime}}{R}+P^{\prime}\right] f^{(k)}-R e^{P} f^{\prime}=Q^{\prime}-\frac{R^{\prime}}{R} Q-P^{\prime}
$$

Substituting $f^{\prime}=g_{1}+z, f^{\prime \prime}=g_{1}^{\prime}+1, f^{(j+1)}=g_{1}^{(j)}(2 \leq j \leq k)$ into (4.4), we get

$$
g_{1}^{(k)}-\left[\frac{R^{\prime}}{R}+P^{\prime}\right] g_{1}^{(k-1)}-R e^{P} f^{\prime} g_{1}=Q^{\prime}-\frac{R^{\prime}}{R} Q-P^{\prime}+z R e^{P} .
$$

Set $h_{1}:=Q^{\prime}-\frac{R^{\prime}}{R} Q-P^{\prime}+z R e^{P}$. Obviously, $h_{1} \not \equiv 0$. Applying Lemma 2.5 to (4.5), we obtain $\bar{\lambda}\left(g_{1}\right)=\bar{\lambda}\left(f^{\prime}-z\right)=\bar{\tau}\left(f^{\prime}\right)=\rho\left(g_{1}\right)=\rho(f)=\infty$.

This completes the proof of Theorem 1.4.

\section{An application}

In 1996, Brück posed the following conjecture (see [22]). 
Conjecture Let $f$ be a nonconstant entire function such that the hyper-order $\sigma_{2}(f)$ of $f$ is not a positive integer and $\sigma_{2}(f)<\infty$. If $f$ and $f^{\prime}$ share a finite value a $C M$, then

$$
\frac{f^{\prime}-a}{f-a}=c
$$

where $c$ is a nonzero constant.

Since then, many results related to the conjecture have been obtained. The conjecture for the cases that $a=0$ has been proved by Brück [22]. Later, Gundersen and Yang [7], Yang [23] proved the following well-known result.

Theorem D Let $f$ be a nonconstant entire function with finite order. Iff and $f^{(k)}$ share a finite value a $C M$, then

$$
\frac{f^{(k)}-a}{f-a}=c
$$

where $c$ is a nonzero constant.

Li and Yi [24] studied the case that an entire function of finite order shares a polynomial with its first derivative. They showed the following result.

Theorem $\mathrm{E}$ Let $f$ be a nonconstant entire function with finite order, and let $Q$ be a nonconstant polynomial. Iff and $f^{\prime}$ share $Q C M$, then

$$
\frac{f^{\prime}-Q}{f-Q}=c
$$

where $c$ is a nonzero constant.

In this section, we will consider the case that an entire function of finite order shares a meromorphic function of order less than 1 with its $k$ th derivative.

Theorem 5.1 Letf be a nonconstant entire function offinite order, $R \not \equiv 0$ be a meromorphic function that has finitely many poles and $\rho(R)<1$, and $k$ be a positive integer. Iff and $f^{(k)}$ share R CM, then

$$
\frac{f^{(k)}-R}{f-R}=c,
$$

where $c$ is a nonzero constant.

Next we prove Theorem 5.1.

Proof Since $f$ is of finite order and $f$ and $f^{(k)}$ share $R$ CM, by the Hadamard factorization theorem for meromorphic functions with finite order, we have

$$
\frac{f^{(k)}-R}{f-R}=e^{P(z)}
$$

where $P(z)$ is a polynomial. 
If $P(z)$ is not a constant. Set $F(z)=f(z)-R(z)$ and $Q(z)=R(z)-R^{(k)}(z)$. Then (5.1) can be rewritten as

$$
F^{(k)}-e^{P(z)} F=Q(z)
$$

Note that $\rho(Q) \leq \rho(R)<1 \leq \operatorname{deg}(P)$. By Corollary 1.2, the order of $F$, and hence of $f$, is infinite. It is a contradiction. Hence $P(z)$ is a constant.

In [25], Li and Yi also proved the following related result.

Theorem $\mathbf{F}$ Let $f$ be a nonconstant entire function of finite order, $R$ be a nonzero rational function that has at least one pole and $k$ be a positive integer. If $f-R$ and $f^{(k)}-R$ share 0 CM, then $f=f^{(k)}$.

From Corollary 1.2, we immediately obtain a corollary which generalizes Theorem F.

Corollary 5.2 Let $f$ be a nonconstant entire function of finite order, $R \not \equiv 0$ be a meromorphic function with finitely many poles (at least one pole) and $\rho(R)<1$ and $k$ be a positive integer. Iff and $f^{(k)}$ share $R C M$, then $f=f^{(k)}$.

\section{Competing interests}

The authors declare that they have no competing interests.

\section{Authors' contributions}

All authors drafted the manuscript, read and approved the final manuscript.

\section{Author details}

${ }^{1}$ Department of Mathematics, Hunan University, Changsha, 410082, P.R. China. ${ }^{2}$ Department of Mathematics, Wuyi University, Jiangmen, 529020, P.R. China. ${ }^{3}$ Department of Mathematics, Zhaoqing University, Zhaoqing, Guangdong 526061, P.R. China.

\section{Acknowledgements}

The authors would like to thank the referee for his/her constructive suggestions. This work was supported by the National Natural Science Foundation of China (Nos. 11126327, 12271090), NSF of Guangdong Province (No. S2011010000735) and the Outstanding Young Innovative Talents Fund of Department of Education of Guangdong (No. 2012LYM0126).

Received: 1 November 2013 Accepted: 3 December 2013 Published: 18 Dec 2013

\section{References}

1. Hayman, WK: Meromorphic Functions. Clarendon, Oxford (1964)

2. Yang, L: Value Distribution Theory. Springer, Berlin (1993)

3. Yang, CC, Yi, HX: Uniqueness Theory of Meromorphic Functions. Kluwer Academic, New York (2003)

4. Chen, ZX: The fixed points and hyper-order of solutions of second order linear differential equations. Acta Math. Sci. 20, 425-432 (2000) (in Chinese)

5. Jank, G, Volkmann, L: Einührung in die Theorie der meromorphen Funktionen mit Anwendungen auf Differentialgleichungen. Birkhäuser, Basel (1985)

6. Markusherish, Al: Theory of Functions of a Complex Variable. Prentice Hall, New York (1965)

7. Gundersen, GG, Yang, LZ: Entire functions that share one value with one or two of their derivatives. J. Math. Anal. Appl. 223, 88-95 (1998)

8. Wang, J, Yi, HX: Fixed points and hyper-order of differential polynomials generated by solutions of differential equation. Complex Var. Theory Appl. 48, 83-94 (2003)

9. Han, Q, Yi, HX: On the uniqueness problems of entire functions and their linear differential polynomials. Kodai Math. J. 30, 66-73 (2007)

10. Lü, F, Liu, K, Yi, HX: Normal families and uniqueness theorem of holomorphic functions. Kodai Math. J. 33, 217-232 (2010)

11. Zhuang, QT, Yang, CC: The Fixed Points and Factorization Theory of Meromorphic Functions. Press in Beijing University, Beijing (1988) (in Chinese)

12. Laine, I, Rieppo, J: Differential polynomials generated by linear differential equations. Complex Var. Theory Appl. 49 , 897-911 (2004) 
13. Chen, ZX, Shon, $\mathrm{KH}$ : On the growth and fixed points of solutions of second order differential equation with meromorphic coefficients. Acta Math. Sin. Engl. Ser. 21, 753-764 (2004)

14. Liu, MS, Zhang, XM: Fixed points of meromorphic solutions of higher order linear differential equations. Ann. Acad. Sci. Fenn., Math. 31, 191-211 (2006)

15. $\mathrm{Xu}, \mathrm{JF}, \mathrm{Yi}, \mathrm{HX}$ : Growth and fixed points of meromorphic solutions of linear differential equations. J. Korean Math. Soc. 46, 747-758 (2009)

16. Gundersen, G: Estimates for the logarithmic derivative of a meromorphic function, plus similar estimates. J. Lond. Math. Soc. 37, 88-104 (1988)

17. Chen, ZX The growth of solutions of differential equation $f^{\prime \prime}+e^{-z} f^{\prime}+Q(z) f=0$. Sci. China Ser. A 31, 775-784 (2001)

18. Chen, ZX: Zeros of meromorphic solutions of higher order linear differential equations. Analysis 14, 425-438 (1999)

19. Wang, J, Laine, I: Growth of solutions of nonhomogeneous linear differential equations. Abstr. Appl. Anal. 2009, Article ID 363927 (2009)

20. Belaïdi, B, Farissi, A: Relation between differential polynomials and small functions. Kyoto J. Math. 50, 453-468 (2010)

21. Xu, JF, Zhang, XB: Some results of meromorphic solutions of second-order linear differential equations. J. Inequal. Appl. 2013, 304 (2013)

22. Brück, R: On entire functions which share one value CM with their first derivatives. Results Math. 78, 655-658 (1971)

23. Yang, LZ: Solution of a differential equation and its applications. Kodai Math. J. 22, 458-464 (1999)

24. Li, XM, Yi, HX: An entire function and its derivatives sharing a polynomial. J. Math. Anal. Appl. 330, 66-79 (2007)

25. Li, XM, Yi, HX: Uniqueness of meromorphic functions sharing a meromorphic function of a smaller order with their derivatives. Ann. Pol. Math. 98, 207-219 (2010)

10.1186/1687-1847-2013-370

Cite this article as: $\mathrm{Xu}$ and Zhang: Growth and fixed points of meromorphic solutions of nonhomogeneous linear

differential equations. Advances in Difference Equations 2013, 2013:370

\section{Submit your manuscript to a SpringerOpen ${ }^{\odot}$ journal and benefit from:}

- Convenient online submission

Rigorous peer review

- Immediate publication on acceptance

- Open access: articles freely available online

- High visibility within the field

- Retaining the copyright to your article 